\title{
Diastereoselective synthesis of 1,1,4-trisubstituted-2,3,4,9- tetrahydrospiro- $\beta$-carbolines via glacial acetic acid catalyzed Pictet - Spengler reaction
}

\author{
Abdullah M. A. Shumaila, ${ }^{\text {a }}$ Vedavati G. Puranik, ${ }^{\text {b }}$ and Radhika S. Kusurkar ${ }^{a, *}$ \\ ${ }^{a}$ Department of Chemistry, University of Pune, Pune, 411007, India \\ ${ }^{b}$ Center for Materials Characterization, National Chemical Laboratory, Pune 411008, India
}

E-mail: rsk@chem.unipune.ac.in

\begin{abstract}
The Pictet-Spengler reaction of substituted tryptamines with cyclic ketones using glacial acetic acid afforded only one diastereomer of unreported 1,1,4-trisubstituted-2,3,4,9-tetrahydrospiro- $\beta$ carbolines. The stereoselectivity in the reaction has been demonstrated using unsymmetrical ketones and single- crystal X-ray analysis of one of the spiro products in the form of base, its sulfate and hydrochloride salts, which indicated the formation of only the $R, R$ diastereomer.
\end{abstract}

Keywords: $1,1,4$-Trisubstituted-2,3,4,9-tetrahydrospiro- $\beta$-carbolines, diastereoselective PictetSpengler reaction, $\beta$-substituted tryptamine, glacial acetic acid

\section{Introduction}

Tetrahydrospiro- $\beta$-carbolines (THS $\beta$ Cs) are important indole alkaloids since they show various biological activities like anticonvulsant, ${ }^{1}$ antispasmodic activity, ${ }^{2}$ GHSR inhibitory activity with $\mathrm{Ki}=60 \mathrm{nM},{ }^{3}$ receptor affinity, ${ }^{4}$ and antidepressant activity. ${ }^{5}$

The Pictet-Spengler reaction ${ }^{6}$ has been extensively studied in the areas of synthesis of different biologically important heterocyclic systems. ${ }^{7}$ Asymmetric Pictet-Spengler reactions have attracted much attention, ${ }^{8-15}$ because it is an important and useful tool to construct chiral synthons containing tetrahydro-isoquinoline or tetrahydro- $\beta$-carboline ( $\mathrm{TH} \beta \mathrm{C})$ structural moieties. Therefore, the main challenge of this cyclization is stereoselectivity and the ratio of isomers. Different conditions, by changing temperature, solvent and acid-catalysts, were studied to improve the selectivity of Pictet-Spengler reaction. ${ }^{16-20}$ Other synthetic strategies used to influence the stereoselectivity of the Pictet-Spengler condensation include the use of chiral catalysts, ${ }^{11}$ chiral auxiliaries, ${ }^{13,21}$ or optically active carbonyl compounds. ${ }^{22}$ Amongst the variously substituted $\mathrm{TH} \beta$ Cs very few reports are available typically for 1,4-disubstituted $\mathrm{TH} \beta \mathrm{Cs}$ and also for 1,1,4-trisubstituted $\mathrm{TH} \beta \mathrm{Cs}^{23-25}$ We now report a highly diastereoselective method 
for synthesizing 1,1,4-trisubstituted THS $\beta$ Cs by the Pictet-Spengler reaction of $\beta$-substituted tryptamine with symmetric and unsymmetric ketones using glacial acetic acid as a catalyst.

\section{Results and Discussion}

Use of silica gel as a solid and mildly acidic catalyst is well reported and is receiving considerable attention from synthetic chemists. ${ }^{26,27}$ In the present study silica gel was used for conjugate addition reaction. Thus, indole and nitro olefin 1 were loaded on silica gel and then heated for $2 \mathrm{~min}$. at $150{ }^{\circ} \mathrm{C}$ to get a solid product in $97 \%$ yield. The structure was shown to be 8 by comparing with the reported ${ }^{27 \mathrm{~d}}$ values. Further reactions of various other nitro olefins $\mathbf{2 - 7}$ and indole by using the same method furnished products 9-14 in good yields and in short time. The results are shown in Scheme 1 and Table 1. Thus, a new efficient method was established for the conjugate addition of indole on nitro olefins.

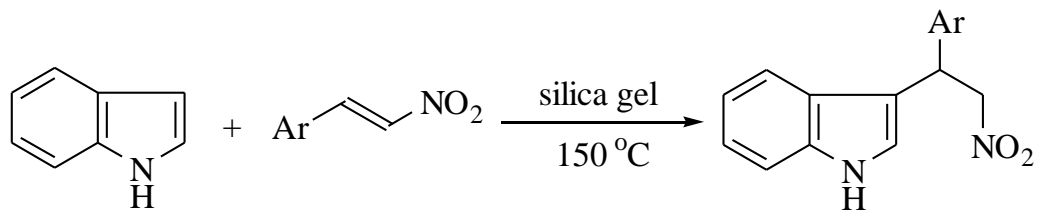

1-7

8-14

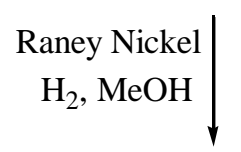

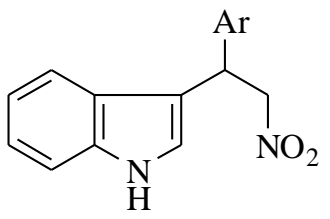

22

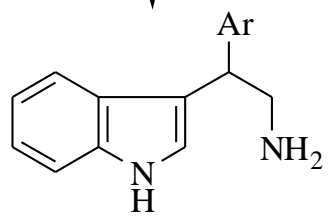

15-21

\begin{tabular}{cccc}
\hline Compd. & Ar & Compd. & Ar \\
\hline $\mathbf{1}, \mathbf{8}, \mathbf{1 5}$ & Phenyl & $\mathbf{5 , 1 2}$ & 4-Nitrophenyl \\
$\mathbf{2 , 9 , \mathbf { 9 }}$ & 4-Methoxyphenyl & $\mathbf{1 9 , 2 2}$ & 4-Aminophenyl \\
$\mathbf{3 , 1 0}, \mathbf{1 7}$ & 3,4-Methylenedioxyphenyl & $\mathbf{6 , 1 3 , 2 0}$ & 2-Thienyl \\
$\mathbf{4 , 1 1}, \mathbf{1 8}$ & 2-Furyl & $\mathbf{7 , 1 4 , \mathbf { 2 1 }}$ & 3,4-Dimethoxyphenyl \\
\hline
\end{tabular}

\section{Scheme 1}

Further reduction of the nitro compound 8-14 using freshly prepared Raney nickel in methanol furnished the $\beta$-phenyltryptamines 15-21. In the case of the nitro compound 12, initial reduction of aromatic nitro group furnished product 22. After continuing the reaction for longer time, both the nitro groups were reduced to give $\mathbf{1 9}$ (Scheme 1). 
To check the catalytic activity of glacial acetic acid for Pictet-Spengler cyclization initially the condensation reaction of amine $\mathbf{1 5}$ and cyclohexanone in the presence of catalytic amount of glacial acetic acid was carried out by refluxing in toluene to furnish a new compound $\mathbf{2 3}$ (Scheme 2, Table 2) in $91 \%$ yield. The spectral and analytical data was consistent with the structure 23 which was confirmed by DEPT experiment. In the ${ }^{13} \mathrm{C} \mathrm{NMR}$, signal at 52.3 was assigned to the spiro carbon $\mathrm{C}_{1}$ which was absent in the DEPT experiment where all protonated carbons are seen. One $\mathrm{CH}$ at 40.8 for $\mathrm{C}_{4}$ and five $-\mathrm{CH}_{2}$ groups with one strong signal at 21.7 were observed in the aliphatic region of the DEPT experiment. This data supported for PictetSpengler cyclization in presence of glacial acetic acid.

Table 1. Time and yield for the Michael addition reactions using nitro olefins 1-7

\begin{tabular}{|c|c|c|c|c|}
\hline Entry & Electrophile & Products & Time (min.) & Yield (\%) \\
\hline 1 & & 8 & 2 & 97 \\
\hline 2 & & 9 & 4 & 91 \\
\hline 3 & & 10 & 5 & 90 \\
\hline 4 & & 11 & 2 & 90 \\
\hline 5 & & 12 & 5 & 87 \\
\hline 6 & 6 & 13 & 2 & 93 \\
\hline 7 & 7 & 14 & 7 & 89 \\
\hline
\end{tabular}

After getting this successful cyclization, similar Pictet-Spengler cyclizations of the amino compounds 16-21 with cyclohexanone and of compounds 15 and 17 with cyclopentanone, furnished eight new 1,1,4-trisubstituted THS $\beta$ Cs 24-29 and 30-31 respectively (Scheme 2, Table 2). Although spiro compounds are known to show dissymmetry, only one racemic product was expected, as the ketones used were symmetric.

To explore the stereoselectivity in the reaction, unsymmetrical ketones such as isatin, $\alpha$ tetralone and 2-methylcyclopentane-1,3-dione were used for the Pictet-Spengler cyclization. Treatment of $\mathbf{1 5}$ with isatin in the presence of a catalytic amount of glacial acetic acid furnished 
a solid product. ${ }^{1} \mathrm{H}$ and ${ }^{13} \mathrm{C}$ NMR indicated it to be a single diastereomer of base 32 (Scheme 3). The stereochemistry of base $\mathbf{3 2}$ was investigated using single crystal X-ray analysis, indicating the $R, R$ configuration at $\mathrm{C}_{1}$ and $\mathrm{C}_{4}$ (Figure 1 ).

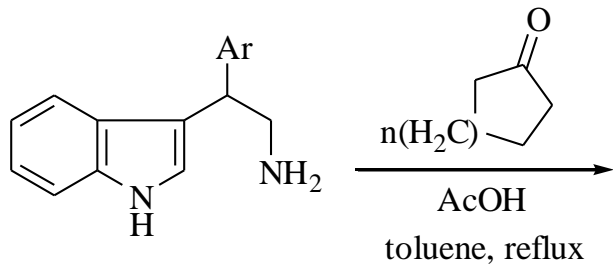

15-21

$n=1$ or 2
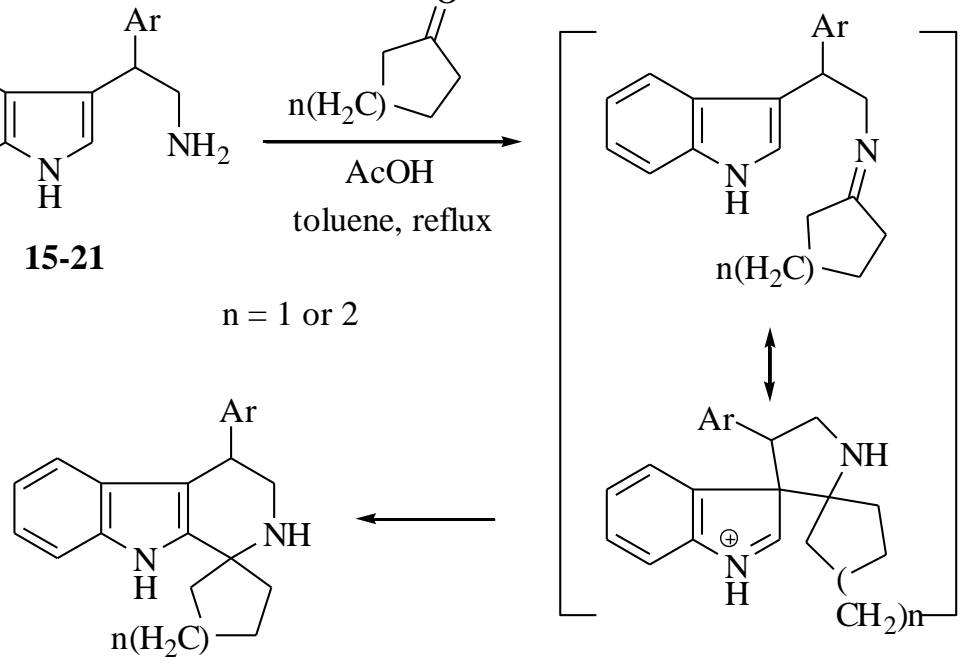

23-31

\begin{tabular}{cccccc}
\hline Compd. & Ar & $\mathbf{n}$ & Compd. & Ar & $\mathbf{n}$ \\
\hline $\mathbf{2 3}$ & Phenyl & 2 & $\mathbf{2 4}$ & 4-methoxyphenyl & 2 \\
$\mathbf{2 5}$ & 3,4-methylenedioxyphenyl & 2 & $\mathbf{2 6}$ & 2-furyl & 2 \\
$\mathbf{2 7}$ & 4-aminophenyl & 2 & $\mathbf{2 8}$ & 2-thienyl & 2 \\
$\mathbf{2 9}$ & 3,4-dimethoxyphenyl & 2 & $\mathbf{3 0}$ & Phenyl & 1 \\
$\mathbf{3 1}$ & 3,4-methylenedioxyphenyl & 1 & & & \\
\hline
\end{tabular}

\section{Scheme 2}

A literature survey revealed that there is a report for the formation of mixture of the two diastereomers of sulfate form of base 32 in a similar Pictet-Spengler condensation using sulfuric acid in water as a catalyst in $46 \%$ yield. In this report, ${ }^{24}$ the major diastereomer was shown to have $R, R$ configuration using $2 \mathrm{D}$ NMR of the mixture without isolating the individual isomers.

However, in the present study using acetic acid as a catalyst we could achieve the formation of only one diastereomer of base $\mathbf{3 2}$ in $88 \%$ yield exclusively and also confirmed the stereochemistry of base $\mathbf{3 2}$ as $R, R$ unambiguously using single crystal X-ray analysis. The sterically preferred spiro transition state (Scheme 3) having the trans arrangement of substituents at $\mathrm{C}_{1}$ and $\mathrm{C}_{4}$ in presence of glacial acetic acid as a mild acidic catalyst explained the exclusive formation of diastereomer 32. 
<smiles>NCC(Br)c1c[nH]c2ccccc12</smiles>

$15,16,17,20$

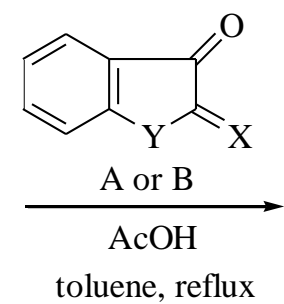

toluene, reflux

$\mathrm{A}: \mathrm{X}=\mathrm{O}, \mathrm{Y}=\mathrm{NH}$

$\mathrm{B}: \mathrm{X}=\mathrm{H} 2, \mathrm{Y}=-\left(\mathrm{CH}_{2}\right)_{2}-$
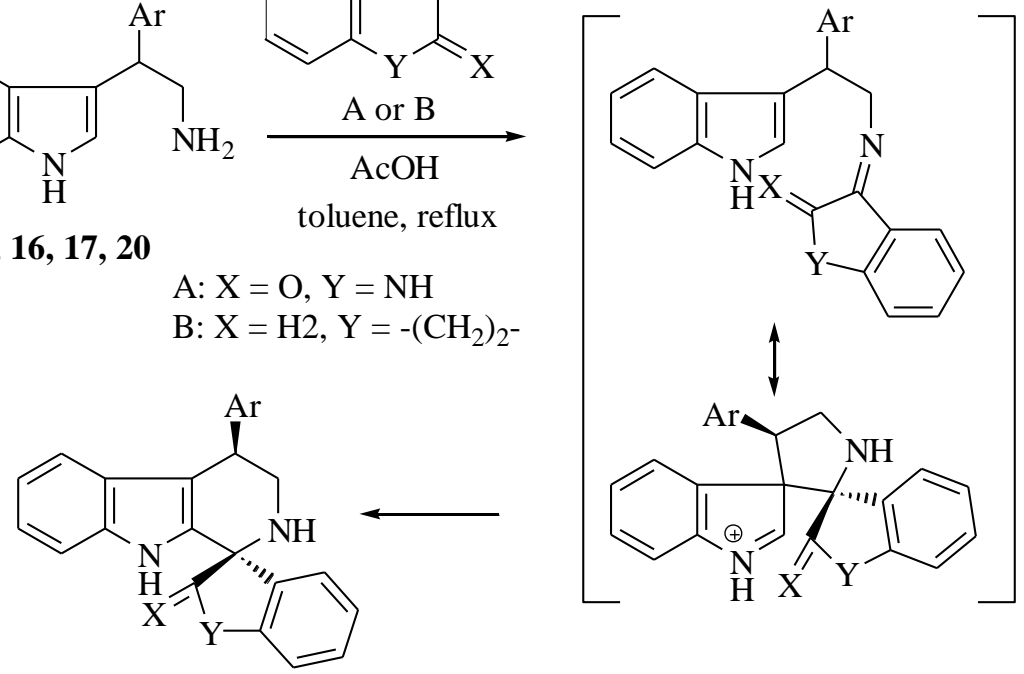

32, 35-39

\begin{tabular}{llll}
\hline Compd. & \multicolumn{1}{c}{ Ar } & X & Y \\
\hline $\mathbf{3 2}$ & Phenyl & $\mathrm{O}$ & $\mathrm{NH}$ \\
$\mathbf{3 5}$ & 4-Methoxyphenyl & $\mathrm{O}$ & $\mathrm{NH}$ \\
$\mathbf{3 6}$ & 3,4-Methylenedioxyphenyl & $\mathrm{O}$ & $\mathrm{NH}$ \\
$\mathbf{3 7}$ & 2-Thienyl & $\mathrm{O}$ & $\mathrm{NH}$ \\
$\mathbf{3 8}$ & Phenyl & $\mathrm{H}_{2}$ & $-\left(\mathrm{CH}_{2}\right)_{2-}$ \\
$\mathbf{3 9}$ & 4-Methoxyphenyl & $\mathrm{H}_{2}$ & $-\left(\mathrm{CH}_{2}\right)_{2-}^{-}$ \\
\hline
\end{tabular}

\section{Scheme 3}

To compare the reported ${ }^{24}$ and the present results, base $\mathbf{3 2}$ was treated with sulfuric acid (conc.) in methanol to furnish the corresponding sulfate $\mathbf{3 3}$ in $95 \%$ yield (Scheme 4). The ${ }^{1} \mathrm{H}$ NMR and ${ }^{13} \mathrm{C}$ NMR data of sulfate $\mathbf{3 3}$ were consistent with that of the reported major isomer.<smiles></smiles>

32

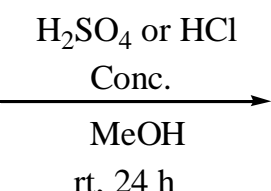

rt, $24 \mathrm{~h}$

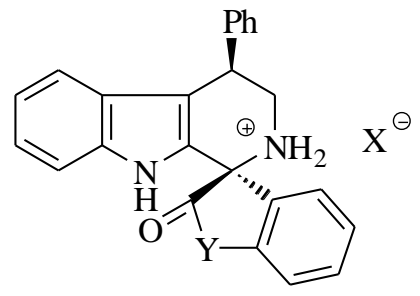

33: $\mathrm{X}=\mathrm{HSO}_{4}$

34: $\mathrm{X}=\mathrm{Cl}$

\section{Scheme 4}


The report $^{2}$ of good biological activity for the hydrochloride of similar base without a substituent at 4-position led us to convert base 32 to the hydrochloride 34 (94\% yield, Scheme 4).

Table 2. Time and yield for Pictet- Spengler reactions using $\beta$-substituted tryptamines

\begin{tabular}{cccccc}
\hline Entry & Compd. & Ketones & Ar & \multicolumn{2}{c}{ THS $\beta$ Cs } \\
& & & & Time (h) & Yield \% \\
\hline 1 & $\mathbf{1 5 , 2 3}$ & Cyclohexanone & Phenyl & 9.5 & 91 \\
2 & $\mathbf{1 6 , 2 4}$ & Cyclohexanone & 4-Methoxyphenyl & 7.5 & 90 \\
3 & $\mathbf{1 7 , 2 5}$ & Cyclohexanone & 3,4-Methylenedioxyphenyl & 7.5 & 89 \\
4 & $\mathbf{1 8 , 2 6}$ & Cyclohexanone & 2-Furyl & 8.5 & 85 \\
5 & $\mathbf{1 9 , 2 7}$ & Cyclohexanone & 4-Aminophenyl & 10.0 & 71 \\
6 & $\mathbf{2 0 , 2 8}$ & Cyclohexanone & 2-Thienyl & 8.5 & 87 \\
7 & $\mathbf{2 1 , 2 9}$ & Cyclohexanone & 3,4-Dimethoxyphenyl & 7.5 & 88 \\
8 & $\mathbf{1 5 , 3 0}$ & Cyclopentanone & Phenyl & 13.5 & 82 \\
9 & $\mathbf{1 7 , 3 1}$ & Cyclopentanone & 3,4-Methylenedioxyphenyl & 11.5 & 79 \\
10 & $\mathbf{1 5 , 3 2}$ & Isatin & Phenyl & 18.5 & 88 \\
11 & $\mathbf{1 6 , 3 5}$ & Isatin & 4-Methoxyphenyl & 18.0 & 84 \\
12 & $\mathbf{1 7 , 3 6}$ & Isatin & 3,4-Methylenedioxyphenyl & 18.5 & 85 \\
13 & $\mathbf{2 0 , 3 7}$ & Isatin & 2-Thienyl & 18.0 & 81 \\
14 & $\mathbf{1 5 , 3 8}$ & $\alpha-T e t r a l o n e$ & Phenyl & 17.5 & 76 \\
15 & $\mathbf{1 6 , 3 9}$ & $\alpha-T e t r a l o n e$ & 4-Methoxyphenyl & 17.5 & 72 \\
\hline
\end{tabular}

In the ${ }^{1} \mathrm{H}$ NMR of salts 33 and 34, the assignments of $\mathrm{C}_{3} \mathrm{H}_{2}$ and $\mathrm{C}_{4} \mathrm{H}$ are different from those of base 32, as shown in Table 3. In both the salts, the protons at $\mathrm{C}_{3}$ are shifted downfield due to the proximity of quaternary nitrogen. The $\mathrm{C}_{4} \mathrm{H}$ appeared as a dd with $J=6.1$ and $12.1 \mathrm{~Hz}$ as $J$ a,e and $J a$,a, respectively showing coupling with two protons at $\mathrm{C}_{3}$.

Table 3. Comparison of ${ }^{1} \mathrm{H}$ NMR spectra of compounds 32, 33 and 34

\begin{tabular}{|c|c|c|c|c|c|c|c|c|c|c|}
\hline \multirow[t]{2}{*}{ Entry } & \multirow[t]{2}{*}{ Assignment } & \multicolumn{2}{|c|}{ Base 32} & \multicolumn{3}{|c|}{ Sulfate 33} & \multicolumn{4}{|c|}{ Hydrochloride 34} \\
\hline & & $\mathrm{C}_{3} \mathrm{H}$ & $\mathrm{C}_{3} \mathrm{H}$ & $\mathrm{C}_{4} \mathrm{H}$ & $\mathrm{C}_{3} \mathrm{H}_{\mathrm{a}}$ & $\mathrm{C}_{3} \mathrm{H}_{\mathrm{e}}$ & $\mathrm{C}_{4} \mathrm{H}$ & $\mathrm{C}_{3} \mathrm{H}_{\mathrm{a}}$ & $\mathrm{C}_{3} \mathrm{H}_{\mathrm{e}}$ & $\mathrm{C}_{4} \mathrm{H}$ \\
\hline 1 & $\begin{array}{l}\text { Chemical } \\
\operatorname{shift}(\delta)\end{array}$ & 3.56 & 3.75 & 4.41 & 4.14 & 4.72 & 3.84 & 4.17 & 4.83 & 3.71 \\
\hline 2 & Multiplicity & dd & dd & $\mathrm{t}$ & $\mathrm{t}$ & $\mathrm{dd}$ & $\mathrm{dd}$ & $\mathrm{t}$ & $\mathrm{dd}$ & $\mathrm{dd}$ \\
\hline 3 & $\mathrm{~J} \mathrm{~Hz}$ & $\begin{array}{l}5.5 \\
13.4\end{array}$ & $\begin{array}{l}6.7 \\
13.4\end{array}$ & 6.7 & 10.7 & $\begin{array}{l}6.1, \\
10.7\end{array}$ & $\begin{array}{l}6.1 \\
12.1\end{array}$ & 11.3 & $\begin{array}{l}6.1, \\
10.6\end{array}$ & $\begin{array}{l}6.1, \\
12.1\end{array}$ \\
\hline
\end{tabular}


This indicated the axial position of $\mathrm{C}_{4} \mathrm{H}$ in both the salts $\mathbf{3 3}$ and $\mathbf{3 4}$. However, in base $\mathbf{3 2} \mathrm{C}_{4} \mathrm{H}$ appeared at 4.41 as a triplet, $J=6.7 \mathrm{~Hz}$, indicating rapid flipping of the nitrogen containing ring in solution.

As compared to the chemical shift of $\mathrm{C}_{4} \mathrm{H}$ in base, this proton, being axial was shifted to up field position in both the salts. In contrast to this the stereochemistry of salts $\mathbf{3 3}$ and $\mathbf{3 4}$ was shown to be same as the base $\mathbf{3 2}$ as $R, R$ using $\mathrm{X}$-ray analysis (Figure 1 and 2). The difference in the two results from ${ }^{1} \mathrm{H}$ NMR and X-ray analysis can be attributed to the solution state where rapid flipping is possible in ${ }^{1} \mathrm{H}$ NMR and rigid solid state in X-ray analysis.

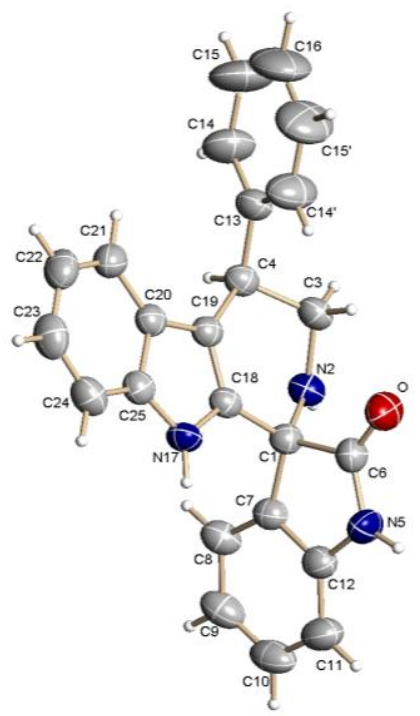

32

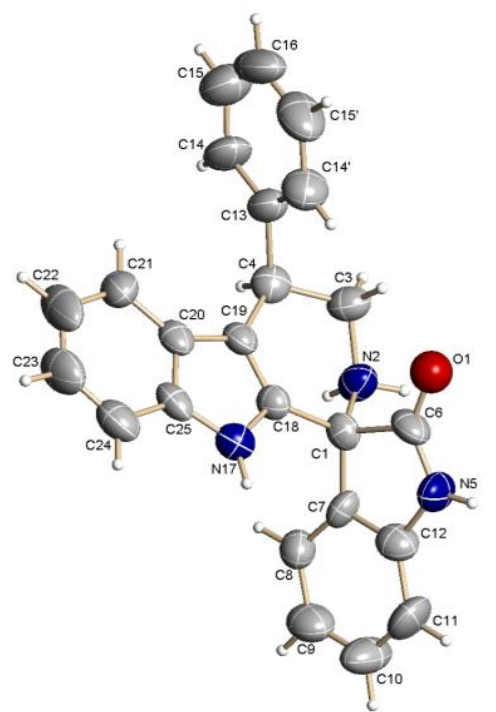

33

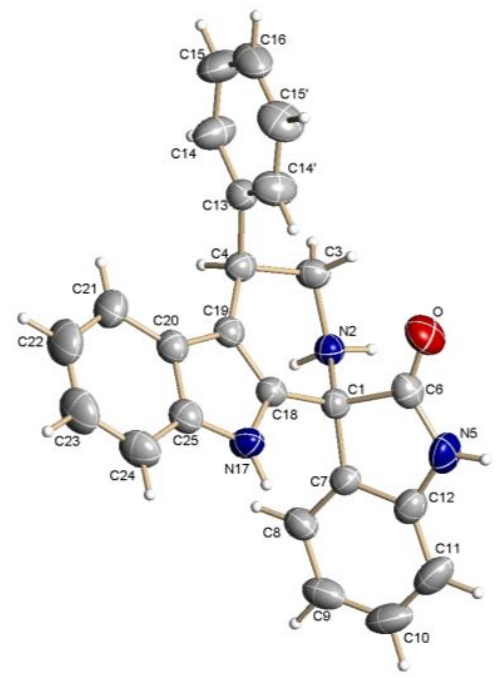

34

Figure 1. ORTEP diagram of base 32, sulfate 33 and hydrochloride $\mathbf{3 4}$ ellipsoids are drawn at $50 \%$ probability.

To generalize the stereoselectivity in the reaction using glacial acetic acid, the substituents at 1 and 4-positions were changed. Treatment of amino compounds 16, 17 and 20 with isatin and 15 and 16 with $\alpha$-tetralone afforded products 35-37 and 38-39 respectively (Scheme 3, Table 2). The similarity in the ${ }^{1} \mathrm{H}$ and ${ }^{13} \mathrm{C}$ NMR of these products, with that of $\mathbf{3 2}$ indicated formation of only one diastereomer in each case.

Subsequently, treatment of amino compound 15 with 2-methylcyclopentane-1,3-dione, under the same conditions led to formation of a new product in $89 \%$ yield. The spectral data was not consistent with the expected structure. Even the attempts for cyclization using strong acid TFA were unsuccessful (Scheme 5). Thus single crystal X-ray analysis was used to assign structure 40 to the new unexpected product (Figure 3). The formation of compound $\mathbf{4 0}$ can be explained by bond isomerization in the imine intermediate to achieve the stable conjugated system. 


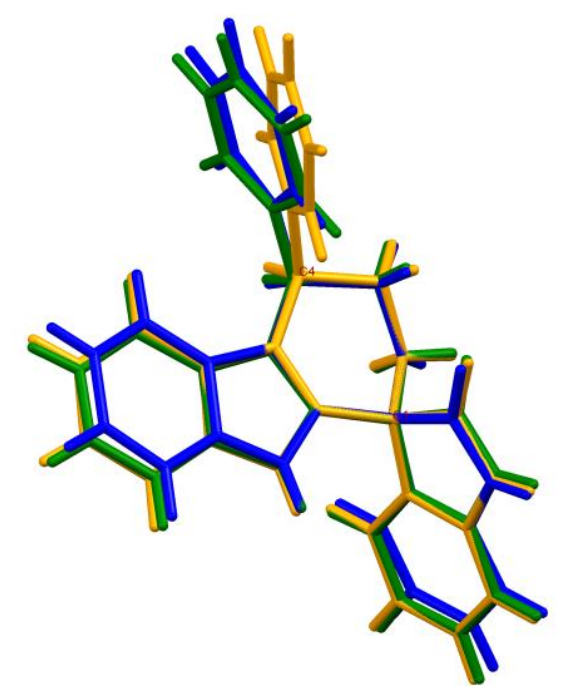

Base 32

Hydrochloride 34

Sulfate 33

Figure 2. X-ray structures overlapping of compounds 32, 33 and 34.

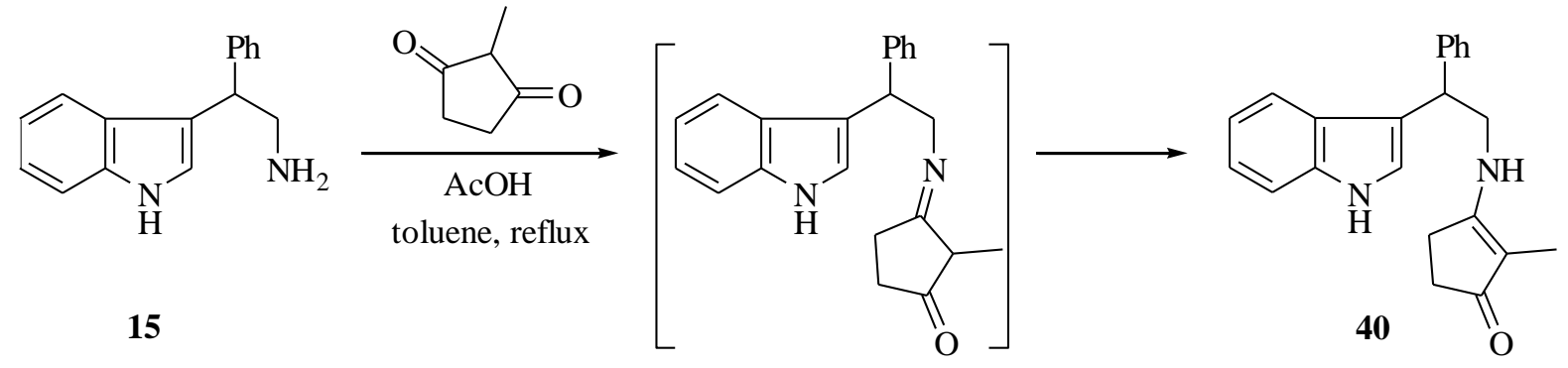

\section{Scheme 5}

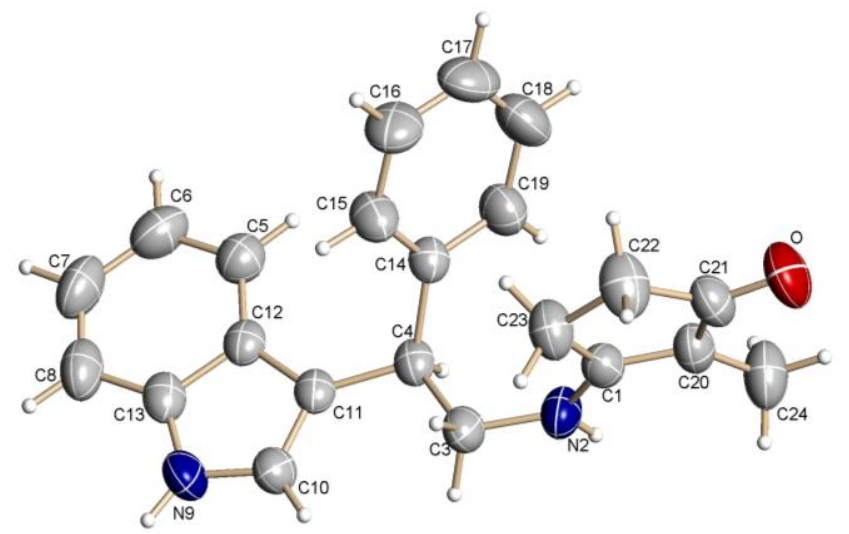

Figure 3. ORTEP diagram of compound 40, ellipsoids is drawn at 50\% probability. 


\section{Conclusions}

In conclusion, catalytic activity of glacial acetic acid in Pictet-Spengler reaction has been demonstrated using symmetric ketones like cyclohexanone and cyclopentanone which furnished new spiro products. Using the same catalyst with unsymmetrical ketones, such as isatin and tetralone, one diastereomer of new THS $\beta$ Cs resulted exclusively. The structures of the base 32, sulfate 33, and hydrochloride $\mathbf{3 4}$ were confirmed using single crystal X-ray analysis, from which the absolute configuration of the products $\mathbf{3 2}, \mathbf{3 3}$ and $\mathbf{3 4}$ was confirmed as $R, R$. Thus, in the present study a diastereoselective and high yielding method for the synthesis of THS $\beta$ Cs was established using glacial acetic acid.

\section{Experimental Section}

General. Melting points recorded are uncorrected. All solvents were of reagent grade and, when necessary, were purified and dried by standard methods. Commercially available glacial acetic acid (from Merck) was used. Reactions and products were routinely monitored by thin layer chromatography (TLC) on silica gel (Kieselgel 60 F254, Merck). Column chromatographic purifications were performed using 60-120 mesh silica gel. Visualization was made with UV light (254 and 365nm) or with an iodine vapor. IR spectra were recorded on Shimadzu 8400 instrument. ${ }^{1} \mathrm{H}$ NMR $(300 \mathrm{MHz})$ and ${ }^{13} \mathrm{C}$ NMR $(75 \mathrm{MHz})$ spectra were recorded on Varian Mercury instrument using TMS as internal standard. ${ }^{1} \mathrm{H}$ NMR peaks expressed as s, bs, d, dd, t, $\mathrm{m}$ correspond to singlet, broad-singlet, doublet, doublet of doublet, triplet, and multiplet, respectively. Mass were recorded on Shimadzu QP 5050. Elemental analyses were recorded on a Vario EL III elemental analyzer instrument.

\section{General procedure for Michael addition of indole on nitro olefins and further reduction to $\beta$-substituted tryptamines}

A mixture of indole $(2.4 \mathrm{mmol})$ and nitro olefin $(2 \mathrm{mmol})$ was loaded on silica gel $(60-120$ mesh, $0.25 \mathrm{~g}$ ) and heated in silica gel bath at $150{ }^{\circ} \mathrm{C}$. After the reaction was complete as judged by TLC, the same silica gel was loaded on a silica-gel column. Chromatographic separation using hexane/ethyl acetate (9:1) furnished the products 8-14. The $\beta$-Substituted tryptamines 1521 were prepared using reported ${ }^{25}$ procedure.

\section{General procedure for the Pictet-Spengler cyclization}

The mixture of the amino compound 15-21, $(2 \mathrm{mmol})$, ketone $(8 \mathrm{mmol})$, and glacial acetic acid (0.1- 0.5 equiv.), was heated at $120{ }^{\circ} \mathrm{C}$ in dry toluene under nitrogen atmosphere in a Dean-Stark apparatus for 7.5-18.5 h. The heating was continued till the full consumption of the amino compound. Completion of the reaction was confirmed by TLC. The reaction mixture was diluted with ethyl acetate, washed with $10 \% \mathrm{NaHCO}_{3}$ and brine. The combined organic layer was dried 
over sodium sulfate and the solvent was evaporated under reduced pressure. The crude product obtained was submitted to column chromatography using hexane/ethyl acetate to give the products 23-32 and 35-40.

4-Phenyl-2,3,4,9-tetrahydrospiro[ $\boldsymbol{\beta}$-carboline-1,1'-cyclohexane] (23). Yield 91\%; yellowish solid; mp $185-187^{\circ} \mathrm{C}$; $\mathrm{R}_{\mathrm{f}}\left(50 \%\right.$ ethyl acetate/hexane) 0.35; IR (KBr): v 3402 (br) cm ${ }^{-1} .{ }^{1} \mathrm{H}$ NMR $\left(300 \mathrm{MHz}, \mathrm{CDCl}_{3}\right) \delta$ 1.48-1.95 (m, 11H, - $\left(\mathrm{C}_{2}\right)_{5^{-}}$, including $\mathrm{N} \underline{\mathrm{H}}$, exchangeable with $\left.\mathrm{D}_{2} \mathrm{O}\right), 3.02$ $\left(\mathrm{dd}, J=5.5,13.5 \mathrm{~Hz}, 1 \mathrm{H}, \mathrm{C}_{3} \underline{\mathrm{H}}\right), 3.39\left(\mathrm{dd}, J=5.2,13.5 \mathrm{~Hz}, 1 \mathrm{H}, \mathrm{C}_{3} \underline{\mathrm{H}}\right), 4.15(\mathrm{t}, J=5.2 \mathrm{~Hz}, 1 \mathrm{H}$, $\mathrm{C}_{4} \underline{\mathrm{H}}$ ), 6.84-6.94 (m, 2H, Ar $\left.\underline{\mathrm{H}}\right), 7.06$ (dt, $\left.J=1.4,8.0 \mathrm{~Hz}, 1 \mathrm{H}, \operatorname{Ar} \underline{\mathrm{H}}\right), 7.11-7.30$ (m, 6H, Ar$\left.\underline{\mathrm{H}}\right), 7.85$ (br s, $1 \mathrm{H}$, exchangeable with $\mathrm{D}_{2} \mathrm{O}, \mathrm{NH}$ of indole ring). ${ }^{13} \mathrm{C} \mathrm{NMR}\left(75 \mathrm{MHz}, \mathrm{CDCl}_{3}\right) \delta 21.4$ (st.), 25.8, 36.4, 37.2, 40.8, 48.5, 52.3, 109.7, 110.5, 119.1, 119.2, 121.3, 126.1, 126.8, 128.1 (st.), 128.2 (st.), 135.4, 142.3, 143.7; $m / z: 316\left(\mathrm{M}^{+}\right)$. Anal. Calcd. for $\mathrm{C}_{22} \mathrm{H}_{24} \mathrm{~N}_{2}$ : C, 83.50; H, 7.64; $\mathrm{N}$, 8.85. Found: C, 83.25; H, 7.89; N, 8.57\%.

4-(4-Methoxyphenyl)-2,3,4,9-tetrahydrospiro[ $\boldsymbol{\beta}$-carboline-1,1'-cyclohexane] (24). Yield 90\%; white solid; mp 187-189 ${ }^{\circ} \mathrm{C}$; $\mathrm{R}_{\mathrm{f}}(60 \%$ ethyl acetate/hexane) 0.29; IR (KBr): $v 3373,3308$ $\mathrm{cm}^{-1}$. ${ }^{1} \mathrm{H}$ NMR $\left(300 \mathrm{MHz}, \mathrm{CDCl}_{3} / \mathrm{DMSO}_{-} d_{6}\right) \delta$ 1.49-2.12 (m, 11H, - $\left(\mathrm{C}_{2}\right)_{5}{ }^{-}$including $\underline{\mathrm{H}}$, exchangeable with $\left.\mathrm{D}_{2} \mathrm{O}\right), 2.95\left(\mathrm{dd}, J=5.8,13.2 \mathrm{~Hz}, 1 \mathrm{H}, \mathrm{C}_{3} \underline{\mathrm{H}}\right), 3.35$ (dd, $J=5.2,13.2 \mathrm{~Hz}, 1 \mathrm{H}$, $\left.\mathrm{C}_{3} \underline{\mathrm{H}}\right), 3.76\left(\mathrm{~s}, 3 \mathrm{H}, \mathrm{OC}_{3}\right), 4.11\left(\mathrm{t}, J=5.2 \mathrm{~Hz}, 1 \mathrm{H}, \mathrm{C}_{4} \underline{\mathrm{H}}\right), 6.76-6.99(\mathrm{~m}, 4 \mathrm{H}, \mathrm{Ar} \underline{\mathrm{H}}), 7.04(\mathrm{t}, J=7.9$

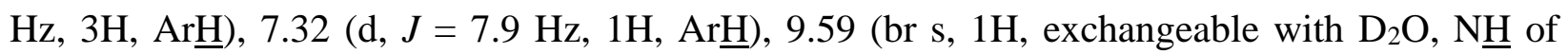
indole ring). ${ }^{13} \mathrm{C} \mathrm{NMR}\left(75 \mathrm{MHz}, \mathrm{CDCl}_{3} / \mathrm{DMSO}-d_{6}\right) \delta 20.5$ (st.), 24.9, 35.0, 35.8, 39.7, 47.8, 51.6, 54.2, 107.9, 110.0, 112.6 (st.), 117.3, 117.8, 119.5, 125.6, 128.1 (st.), 134.9, 135.2, 141.9, 156.8; m/z: $346\left(\mathrm{M}^{+}\right)$; Anal. Calcd. for $\mathrm{C}_{23} \mathrm{H}_{26} \mathrm{~N}_{2} \mathrm{O}$ : C, 79.73; H, 7.56; N, 8.09. Found: C, 79.51; H, 7.69; N; 7.85\%.

4-(3,4-Methylenedioxyphenyl)-2,3,4,9-tetrahydrospiro[ $\beta$-carboline-1,1'-cyclohexane] (25). Yield 89\%; white solid; mp 201-203 ${ }^{\circ} \mathrm{C}$; $\mathrm{R}_{\mathrm{f}}$ (70\% ethyl acetate/hexane) 0.40; IR (KBr): $v 3412$ (br) $\mathrm{cm}^{-1} .{ }^{1} \mathrm{H}$ NMR $\left(300 \mathrm{MHz}, \mathrm{CDCl}_{3}\right) \delta 1.55-2.16\left(\mathrm{~m}, 11 \mathrm{H},-\left(\mathrm{C}_{2}\right)_{5}-\right.$ including $\mathrm{N} \underline{\mathrm{H}}$, exchangeable with $\left.\mathrm{D}_{2} \mathrm{O}\right), 2.98\left(\mathrm{dd}, J=5.5,13.5 \mathrm{~Hz}, 1 \mathrm{H}, \mathrm{C}_{3} \underline{\mathrm{H}}\right), 3.38(\mathrm{dd}, J=5.0,13.5 \mathrm{~Hz}, 1 \mathrm{H}$, $\left.\mathrm{C}_{3} \underline{\mathrm{H}}\right), 4.09$ (t, $\left.J=5.2 \mathrm{~Hz}, 1 \mathrm{H}, \mathrm{C}_{4} \underline{\mathrm{H}}\right), 5.89$ (d, $\left.J=2.2 \mathrm{~Hz}, 2 \mathrm{H}, \mathrm{OC}_{2} \mathrm{O}\right), 6.63(\mathrm{~d}, J=9.1 \mathrm{~Hz}, 2 \mathrm{H}$, $\operatorname{Ar} \underline{\mathrm{H}}), 6.71(\mathrm{~d}, J=7.9 \mathrm{~Hz}, 1 \mathrm{H}, \operatorname{Ar} \underline{\mathrm{H}}), 6.90(\mathrm{t}, J=7.7 \mathrm{~Hz}, 1 \mathrm{H}, \operatorname{Ar} \underline{\mathrm{H}}), 7.00(\mathrm{~d}, J=7.4 \mathrm{~Hz}, 1 \mathrm{H}$, $\operatorname{Ar} \underline{\mathrm{H}}), 7.09$ (t, $J=7.4 \mathrm{~Hz}, 1 \mathrm{H}, \operatorname{Ar} \underline{\mathrm{H}}), 7.30$ (d, $J=7.9 \mathrm{~Hz}, 1 \mathrm{H}, \operatorname{Ar} \underline{\mathrm{H}}), 7.83$ (br s, 1H, exchangeable with $\mathrm{D}_{2} \mathrm{O}, \mathrm{NH}$ of indole ring); ${ }^{13} \mathrm{C} \mathrm{NMR}\left(75 \mathrm{MHz}, \mathrm{CDCl}_{3} / \mathrm{DMSO}-d_{6}\right) \delta 20.4$ (st.), 24.8, 34.7, 35.7, 39.6, 47.6, 51.5, 99.6, 106.8, 107.4, 107.6, 109.9, 117.3, 117.7, 119.5, 119.9, 125.4, 134.9, 137.2, 141.8, 144.6, 146.3; m/z: $360\left(\mathrm{M}^{+}\right)$. Anal. Calcd. for $\mathrm{C}_{23} \mathrm{H}_{24} \mathrm{~N}_{2} \mathrm{O}_{2}: \mathrm{C}, 76.64 ; \mathrm{H}, 6.71$; N,7.77. Found: C, 76.39; H, 6.58; N, 7.98\%.

4-(2-Furyl)-2,3,4,9-tetrahydrospiro[ $\boldsymbol{\beta}$-carboline-1,1'-cyclohexane] (26). Yield 85\%; white solid; mp 260-262 ${ }^{\circ} \mathrm{C}$; $\mathrm{R}_{\mathrm{f}}\left(50 \%\right.$ ethyl acetate/hexane) 0.41; IR (KBr): $v 3300$ (br) cm ${ }^{-1} .{ }^{1} \mathrm{H}$ NMR $\left(300 \mathrm{MHz}, \mathrm{CDCl}_{3}\right) \delta 1.39-1.99\left(\mathrm{~m}, 11 \mathrm{H},-\left(\mathrm{C}_{2}\right)_{5}\right.$ - including $\mathrm{N} \underline{\mathrm{H}}$, exchangeable with $\left.\mathrm{D}_{2} \mathrm{O}\right), 3.18$

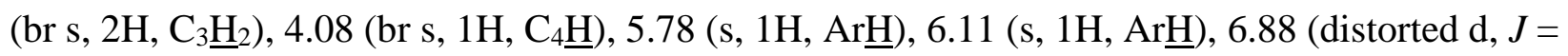
$7.2 \mathrm{~Hz}, 1 \mathrm{H}, \operatorname{Ar} \underline{\mathrm{H}}), 6.98(\mathrm{t}, J=7.4 \mathrm{~Hz}, 1 \mathrm{H}, \operatorname{Ar} \underline{\mathrm{H}}), 7.12(\mathrm{~m}, 3 \mathrm{H}, \operatorname{Ar} \underline{\mathrm{H}}), 7.78$ (s, 1H, exchangeable with $\mathrm{D}_{2} \mathrm{O}, \mathrm{N} \underline{\mathrm{H}}$ of indole ring). ${ }^{13} \mathrm{C} \mathrm{NMR}\left(75 \mathrm{MHz}, \mathrm{CDCl}_{3}\right) \delta 21.3,21.4,25.8,33.8,35.4,37.9$, 
44.4, 52.3, 106.6, 107.8, 109.9, 110.6, 118.6, 119.3, 121.3, 126.8, 135.2, 141.3, 142.1, 156.9; $m / z: 306\left(\mathrm{M}^{+}\right)$. Anal. Calcd. for $\mathrm{C}_{20} \mathrm{H}_{22} \mathrm{~N}_{2} \mathrm{O}: \mathrm{C}, 78.40 ; \mathrm{H}, 7.24 ; \mathrm{N}, 9.14$. Found: C, 78.19; H, $7.07 ; \mathrm{N}, 9.02 \%$.

4-(4-Aminophenyl)-2,3,4,9-tetrahydrospiro[ $\boldsymbol{\beta}$-carboline-1,1'-cyclohexane] (27). Yield 71\%; white solid; $\mathrm{mp} 224-226{ }^{\circ} \mathrm{C}$; $\mathrm{R}_{\mathrm{f}}$ (70\% ethyl acetate/hexane) 0.13; IR (KBr): $v$ 3458, 3346, 3225 (br) $\mathrm{cm}^{-1} .{ }^{1} \mathrm{H}$ NMR $\left(300 \mathrm{MHz}, \mathrm{DMSO}-d_{6}\right) \delta$ 1.43-2.05 (m, 13H, - $\left(\mathrm{C}_{2}\right)_{5}$ - including $\mathrm{N} \underline{\mathrm{H}}, \mathrm{NH}_{2}$, exchangeable with $\left.\mathrm{D}_{2} \mathrm{O}\right), 2.75\left(\mathrm{dd}, J=7.4,13.2 \mathrm{~Hz}, 1 \mathrm{H}, \mathrm{C}_{3} \underline{\mathrm{H}}\right), 3.15(\mathrm{dd}, J=7.7,13.2 \mathrm{~Hz}, 1 \mathrm{H}$, $\mathrm{C}_{3} \underline{\mathrm{H}}$ ), 3.90 (br s, overlapped on DMSO signal, $1 \mathrm{H}, \mathrm{C}_{4} \underline{\mathrm{H}}$ ), 6.45 (d, $J=7.9 \mathrm{~Hz}, 2 \mathrm{H}, \mathrm{Ar} \underline{\mathrm{H}}$ ), 6.59-

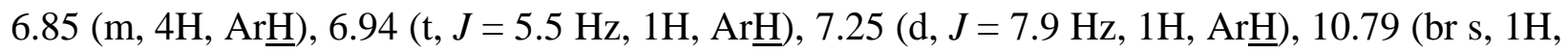
exchangeable with $\mathrm{D}_{2} \mathrm{O}, \mathrm{NH}$ of indole ring). ${ }^{13} \mathrm{C}$ NMR $\left(75 \mathrm{MHz}, \mathrm{DMSO}-d_{6}\right) \delta 20.9,21.5,25.5$, 35.7 (st.), 40.3, 48.5, 52.2, 109.3, 110.8, 113.9 (st.), 117.8, 118.6, 120.0, 126.3, 128.4 (st.), 131.2, 135.5, 142.4, 146.6; m/z: 331 $\left(\mathrm{M}^{+}\right)$. Anal. Calcd. for $\mathrm{C}_{22} \mathrm{H}_{25} \mathrm{~N}_{3}$ : C, 79.72; H, 7.60; N, 12.68 . Found: C, 79.49; H, 7.35; N, 12.39\%.

4-(2-Thienyl)-2,3,4,9-tetrahydrospiro[ $\boldsymbol{\beta}$-carboline-1,1'-cyclohexane] (28). Yield 87\%; brown solid; mp 78-80 ${ }^{\circ} \mathrm{C}$; $\mathrm{R}_{\mathrm{f}}\left(50 \%\right.$ ethyl acetate/hexane) 0.53; IR (KBr): $v 3410,3273 \mathrm{~cm}^{-1} .{ }^{1} \mathrm{H}$ NMR $\left(300 \mathrm{MHz}, \mathrm{CDCl}_{3}\right) \delta 1.40-1.89\left(\mathrm{~m}, 11 \mathrm{H},-\left(\mathrm{C}_{2}\right)_{5}\right.$ - including $\mathrm{NH}$, exchangeable with $\left.\mathrm{D}_{2} \mathrm{O}\right), 3.05$ $\left(\mathrm{dd}, J=3.3,13.2 \mathrm{~Hz}, 1 \mathrm{H}, \mathrm{C}_{3} \underline{\mathrm{H}}\right), 3.30\left(\mathrm{dd}, J=3.8,13.2 \mathrm{~Hz}, 1 \mathrm{H}, \mathrm{C}_{3} \underline{\mathrm{H}}\right), 4.30$ (distorted $\mathrm{t}, J=3.8$ $\left.\mathrm{Hz}, 1 \mathrm{H}, \mathrm{C}_{4} \underline{\mathrm{H}}\right), 6.68(\mathrm{~d}, J=2.8 \mathrm{~Hz}, 1 \mathrm{H}, \operatorname{Ar} \underline{\mathrm{H}}), 6.78(\mathrm{t}, J=3.8 \mathrm{~Hz}, 1 \mathrm{H}, \operatorname{Ar} \underline{\mathrm{H}}), 6.87$ (t, $J=7.7 \mathrm{~Hz}$, $1 \mathrm{H}, \operatorname{Ar} \underline{\mathrm{H}}), 7.0$ (d, $J=5.5 \mathrm{~Hz}, 2 \mathrm{H}, \operatorname{Ar} \underline{\mathrm{H}}), 7.13$ (d, $J=7.9 \mathrm{~Hz}, 1 \mathrm{H}, \operatorname{Ar} \underline{\mathrm{H}}), 7.19$ (d, $J=7.9 \mathrm{~Hz}, 1 \mathrm{H}$, $\mathrm{Ar} \underline{\mathrm{H}}$ ), 7.73 (br s, $1 \mathrm{H}$, exchangeable with $\mathrm{D}_{2} \mathrm{O}, \mathrm{NH}$ of indole ring). ${ }^{13} \mathrm{C} \mathrm{NMR}\left(75 \mathrm{MHz}, \mathrm{CDCl}_{3}\right) \delta$ 21.3, 21.4, 25.9, 35.5 (st.), 37.9, 48.5, 52.3, 110.2, 110.6, 118.7, 119.3, 121.4, 123.4, 124.3, 126.5, 126.8, 135.3, 141.8, 148.5; m/z: $322\left(\mathrm{M}^{+}\right)$. Anal. Calcd. for $\mathrm{C}_{20} \mathrm{H}_{22} \mathrm{~N}_{2} \mathrm{~S}: \mathrm{C}, 74.49 ; \mathrm{H}, 6.88$; N 8.69. Found: C, 74.18; H, 6.56; N 8.42\%.

4-(3,4-Dimethoxyphenyl)-2,3,4,9-tetrahydrospiro[ $\beta$-carboline-1,1'-cyclohexane] (29). Yield 88\%; white solid; $\mathrm{mp} 260-262^{\circ} \mathrm{C}$; $\mathrm{R}_{\mathrm{f}}$ (70\% ethyl acetate/hexane) 0.27 ; IR (KBr): $v 3358$ (br) cm ${ }^{1}$. ${ }^{1} \mathrm{H}$ NMR $\left(300 \mathrm{MHz}, \mathrm{CDCl}_{3}\right) \delta 1.49-1.98\left(\mathrm{~m}, 11 \mathrm{H},-\left(\mathrm{C}_{2}\right)_{5}\right.$ - including $\mathrm{N}$, exchangeable with $\left.\mathrm{D}_{2} \mathrm{O}\right), 3.01\left(\mathrm{dd}, J=5.2,13.5 \mathrm{~Hz}, 1 \mathrm{H}, \mathrm{C}_{3} \underline{\mathrm{H}}\right), 3.35\left(\mathrm{dd}, J=4.7,13.5 \mathrm{~Hz}, 1 \mathrm{H}, \mathrm{C}_{3} \underline{\mathrm{H}}\right), 3.77(\mathrm{~s}, 3 \mathrm{H}$, $\left.\mathrm{OC}_{3}\right), 3.83\left(\mathrm{~s}, 3 \mathrm{H}, \mathrm{OC}_{3}\right), 4.08\left(\mathrm{t}, J=4.7 \mathrm{~Hz}, 1 \mathrm{H}, \mathrm{C}_{4} \underline{\mathrm{H}}\right), 6.63(\mathrm{~d}, J=7.9 \mathrm{~Hz}, 1 \mathrm{H}, \mathrm{Ar} \underline{\mathrm{H}}), 6.72-$ $6.75(\mathrm{~m}, 2 \mathrm{H}, \operatorname{Ar} \underline{\mathrm{H}}), 6.89$ (t, $J=7.4 \mathrm{~Hz}, 1 \mathrm{H}, \operatorname{Ar} \underline{\mathrm{H}}), 6.98$ (d, $J=7.7 \mathrm{~Hz}, 1 \mathrm{H}, \operatorname{Ar} \underline{\mathrm{H}}), 7.08$ (t, $J=7.7$ $\mathrm{Hz}, 1 \mathrm{H}, \operatorname{Ar} \underline{\mathrm{H}}), 7.30(\mathrm{~d}, J=7.9 \mathrm{~Hz}, 1 \mathrm{H}, \operatorname{Ar} \underline{\mathrm{H}}), 7.82$ (br s, $1 \mathrm{H}$, exchangeable with $\mathrm{D}_{2} \mathrm{O}, \mathrm{N} \underline{\mathrm{H}}$ of indole ring). ${ }^{13} \mathrm{C} \mathrm{NMR}\left(75 \mathrm{MHz}, \mathrm{CDCl}_{3} / \mathrm{DMSO}-d_{6}\right) \delta 20.4$ (st.), 24.9, 34.8, 36.1, 39.7, 47.7, 51.4, 54.8 (st.), 107.7, 109.9, 110.0, 110.6, 117.2, 117.6, 119.1, 119.4, 125.6, 134.7, 136.0, 142.0, 146.1, 147.5; m/z: $376\left(\mathrm{M}^{+}\right)$. Anal. Calcd. for $\mathrm{C}_{24} \mathrm{H}_{28} \mathrm{~N}_{2} \mathrm{O}_{2}: \mathrm{C}, 76.56 ; \mathrm{H}, 7.50 ; \mathrm{N}, 7.44$. Found: C, 76.75; H, 7.65; N, 7.23\%.

4-Phenyl-2,3,4,9-tetrahydrospiro[ $\boldsymbol{\beta}$-carboline-1,1'-cyclopentane] (30). Yield 82\%; brown solid; mp 133-135 ${ }^{\circ} \mathrm{C}$; $\mathrm{R}_{\mathrm{f}}\left(50 \%\right.$ ethyl acetate/hexane) 0.38; IR (KBr): $v 3425$ (br) cm ${ }^{-1} .{ }^{1} \mathrm{H}$ NMR $\left(300 \mathrm{MHz}, \mathrm{CDCl}_{3}\right) \delta 1.81-2.15\left(\mathrm{~m}, 9 \mathrm{H},-\left(\mathrm{C}_{2}\right)_{4^{-}}\right.$, including $\mathrm{NH}$, exchangeable with $\left.\mathrm{D}_{2} \mathrm{O}\right), 3.03$ $\left(\mathrm{dd}, J=5.5,13.5 \mathrm{~Hz}, 1 \mathrm{H}, \mathrm{C}_{3} \underline{\mathrm{H}}\right), 3.43\left(\mathrm{dd}, J=5.0,13.5 \mathrm{~Hz}, 1 \mathrm{H}, \mathrm{C}_{3} \underline{\mathrm{H}}\right), 4.14(\mathrm{t}, J=5.0 \mathrm{~Hz}, 1 \mathrm{H}$,

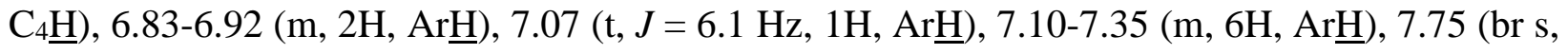
$1 \mathrm{H}$, exchangeable with $\mathrm{D}_{2} \mathrm{O}, \mathrm{N} \underline{\mathrm{H}}$ of indole ring). ${ }^{13} \mathrm{C} \mathrm{NMR}\left(75 \mathrm{MHz}, \mathrm{CDCl}_{3}\right) \delta 25.1$ (st.), 40.2, 
40.7, 40.9, 50.0, 61.8, 110.5 (st.), 119.1, 119.2, 121.4, 126.2, 126.8, 128.1 (st.), 128.2 (st.), 135.6, 140.5, 143.5; m/z: $302\left(\mathrm{M}^{+}\right)$. Anal. Calcd. for $\mathrm{C}_{21} \mathrm{H}_{22} \mathrm{~N}_{2}$ : C, 83.40; H, 7.33; N, 9.26. Found: C, $83.18 ; \mathrm{H}, 7.59 ; \mathrm{N}, 9.05 \%$.

4-(3,4-Methylenedioxyphenyl)-2,3,4,9-tetrahydrospiro[ $\beta$-carboline-1,1'-cyclopentane] (31). Yield 79\%; white solid; mp 245-247 ${ }^{\circ} \mathrm{C}$; $\mathrm{R}_{\mathrm{f}}$ (70\% ethyl acetate/hexane) 0.44 ; IR (KBr): $v 3412$ (br) $\mathrm{cm}^{-1} .{ }^{1} \mathrm{H} \mathrm{NMR}\left(300 \mathrm{MHz}, \mathrm{CDCl}_{3}\right) \delta 1.42-2.01 \quad\left(\mathrm{~m}, 9 \mathrm{H},-\left(\mathrm{C}_{2}\right)_{4} 4^{-}\right.$including $\mathrm{N} \underline{\mathrm{H}}$, exchangeable with $\left.\mathrm{D}_{2} \mathrm{O}\right), 2.90\left(\mathrm{dd}, J=5.5,13.2 \mathrm{~Hz}, 1 \mathrm{H}, \mathrm{C}_{3} \underline{\mathrm{H}}\right), 3.26$ (distorted $\mathrm{t}, J=5.0 \mathrm{~Hz}, 1 \mathrm{H}$, $\mathrm{C}_{3} \underline{\mathrm{H}}$ ), 3.98 (br d, $\left.J=4.4 \mathrm{~Hz}, 1 \mathrm{H}, \mathrm{C}_{4} \underline{\mathrm{H}}\right), 5.77\left(\mathrm{~d}, J=5.5 \mathrm{~Hz}, 2 \mathrm{H}, \mathrm{OC}_{2} \mathrm{O}\right), 6.45-6.65$ (m, $3 \mathrm{H}$, $\operatorname{Ar} \underline{\mathrm{H}}), 6.75-7.05(\mathrm{~m}, 3 \mathrm{H}, \operatorname{Ar} \underline{\mathrm{H}}), 7.15(\mathrm{t},=J 8.2 \mathrm{~Hz}, 1 \mathrm{H}, \mathrm{Ar} \underline{\mathrm{H}}), 7.76$ (br s, 1H, exchangeable with $\mathrm{D}_{2} \mathrm{O}, \mathrm{N} \underline{\mathrm{H}}$ of indole ring). ${ }^{13} \mathrm{C} \mathrm{NMR}\left(75 \mathrm{MHz}, \mathrm{CDCl}_{3}\right) \delta 25.0$ (st.), 40.1, 40.3, 40.8, 50.0, 61.7, 100.7, 108.0, 108.4, 110.4, 110.5, 119.0, 119.2, 121.0, 121.4, 126.7, 135.6, 137.7, 140.4, 145.8, 147.4; m/z: $346\left(\mathrm{M}^{+}\right)$. Anal. Calcd. for $\mathrm{C}_{22} \mathrm{H}_{22} \mathrm{~N}_{2} \mathrm{O}_{2}$ : C, 76.28; H, 6.40; N 8.09. Found: C, 76.06; $\mathrm{H}, 6.61 ; \mathrm{N}, 7.92 \%$.

4-Phenyl-2,3,4,9-tetrahydrospiro[ $\boldsymbol{\beta}$-carboline-1,3'-indol $]-2^{\prime}\left(\mathbf{1}^{\prime} \boldsymbol{H}\right)$-one $\quad(32) . \quad$ Yield $\quad 88 \%$; Colorless crystal; mp $199-201^{\circ} \mathrm{C}$; $\mathrm{R}_{\mathrm{f}}(60 \%$ ethyl acetate/hexane) 0.50; IR (KBr): v 3296 (br.), 3263, $1722 \mathrm{~cm}^{-1} .{ }^{1} \mathrm{H}$ NMR $\left(300 \mathrm{MHz}, \mathrm{CDCl}_{3}\right) \delta 1.69$ (br s, $1 \mathrm{H}$, exchangeable with $\mathrm{D}_{2} \mathrm{O}, \mathrm{N} \underline{\mathrm{H}}$ ), $3.56\left(\mathrm{dd}, J=5.5,13.4 \mathrm{~Hz}, 1 \mathrm{H}, \mathrm{C}_{3} \underline{\mathrm{H}}\right), 3.75\left(\mathrm{dd}, J=6.7,13.4 \mathrm{~Hz}, 1 \mathrm{H}, \mathrm{C}_{3} \underline{\mathrm{H}}\right), 4.41(\mathrm{t}, J=6.7 \mathrm{~Hz}$,

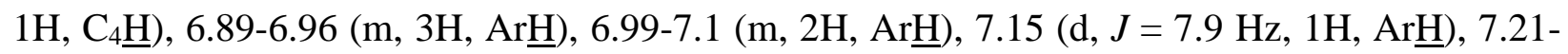

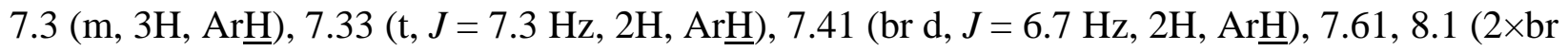
$\mathrm{s}, 2 \mathrm{H}$, exchangeable with $\mathrm{D}_{2} \mathrm{O}, 2 \times \mathrm{NH}$ of isatyl ring and indole ring). ${ }^{13} \mathrm{C} \mathrm{NMR}(75 \mathrm{MHz}$, DMSO- $\left.d_{6}\right) \delta 40.3,48.3,61.8,110.9,111.1,114.0,119.2,119.5,122.1,123.0,124.6,126.1$, 126.5, 128.1, 128.3 (st.), 128.4 (st.), 129.8, 131.0, 136.3, 140.7, 142.6, 178.8; m/z: $365\left(\mathrm{M}^{+}\right)$. Anal. Calcd. for $\mathrm{C}_{24} \mathrm{H}_{19} \mathrm{~N}_{3} \mathrm{O}: \mathrm{C}, 78.88 ; \mathrm{H}, 5.24 ; \mathrm{N}, 11.50$. Found: C, 78.65; H, 5.39; N, 11.34\%.

4-(4-Methoxyphenyl)-2,3,4,9-tetrahydrospiro[ $\beta$-carboline-1,3'-indol]-2'(1'H)-one (35). Yield 84\%, white solid; mp 280-281 ${ }^{\circ} \mathrm{C}$; $\mathrm{R}_{\mathrm{f}}$ (70\% ethyl acetate/hexane) 0.47; IR (KBr): v 3610, 3342, 3286, $1732 \mathrm{~cm}^{-1} .{ }^{1} \mathrm{H}$ NMR (300 MHz, DMSO-d6) $\delta 2.50\left(\mathrm{~s}, 1 \mathrm{H}\right.$, exchangeable with $\mathrm{D}_{2} \mathrm{O}, \mathrm{N} \underline{\mathrm{H}}$ ), 3.26 (distorted t, $J=5.0 \mathrm{~Hz}, 1 \mathrm{H}, \mathrm{C}_{3} \underline{\mathrm{H}}$ ), 3.50 (distorted t, $J=6.6 \mathrm{~Hz}, 1 \mathrm{H}, \mathrm{C}_{3} \underline{\mathrm{H}}$ ), 3.73 (s, $3 \mathrm{H}$, $\left.\mathrm{OC}_{3}\right), 4.33\left(\mathrm{t}, J=6.1 \mathrm{~Hz}, 1 \mathrm{H}, \mathrm{C}_{4} \underline{\mathrm{H}}\right), 6.63-6.78(\mathrm{~m}, 2 \mathrm{H}, \mathrm{Ar} \underline{\mathrm{H}}), 6.82-7.01(\mathrm{~m}, 5 \mathrm{H}, \mathrm{Ar} \underline{\mathrm{H}}), 7.15$ (t, $J$ $=7.9 \mathrm{~Hz}, 2 \mathrm{H}, \mathrm{Ar} \underline{\mathrm{H}}), 7.20-7.33(\mathrm{~m}, 3 \mathrm{H}, \mathrm{Ar} \underline{\mathrm{H}}), 10.53,10.57\left(2 \times \mathrm{s}, 2 \mathrm{H}\right.$, exchangeable with $\mathrm{D}_{2} \mathrm{O}$, $2 \times \mathrm{NH}$ of isatyl ring and indole ring). ${ }^{13} \mathrm{C} \mathrm{NMR}\left(75 \mathrm{MHz}, \mathrm{DMSO}-d_{6}\right) \delta 39.9,48.5,54.9,61.0$, $109.7,111.0,113.0,113.4$ (st.), 118.0, 118.6, 120.7, 121.6, 124.4, 125.7, 129.0 (st.), 132.3, 132.5, 135.5, 136.1, 142.2, 157.5, 177.9; $\mathrm{m} / \mathrm{z}: 395\left(\mathrm{M}^{+}\right)$; Anal. Calc. for $\mathrm{C}_{25} \mathrm{H}_{21} \mathrm{~N}_{3} \mathrm{O}_{2}: \mathrm{C}, 75.93$; H, 5.35; N, 10.63. Found: C, 75.75; H, 5.09; N 10.45\%.

\section{4-(3,4-Methylenedioxyphenyl)-2,3,4,9-tetrahydrospiro[ $\beta$-carboline-1,3'-indol $]-2^{\prime}\left(1^{\prime} H\right)$-one}

(36). Yield 85\%; yellowish solid; mp 266-268 ${ }^{\circ} \mathrm{C}$; $\mathrm{R}_{\mathrm{f}}(70 \%$ ethyl acetate/hexane) 0.46 ; IR (KBr): $v 3398$ (br), 3288, $1730 \mathrm{~cm}^{-1}$. ${ }^{1} \mathrm{H}$ NMR (300 MHz, DMSO-d6) $\delta 3.03$ (s, 1H, exchangeable with $\left.\mathrm{D}_{2} \mathrm{O}, \mathrm{N} \underline{\mathrm{H}}\right), 3.28\left(\mathrm{dd}, J=5.3,12.9 \mathrm{~Hz}, 1 \mathrm{H}, \mathrm{C}_{3} \underline{\mathrm{H}}\right), 3.45\left(\mathrm{dd}, J=6.6,12.9 \mathrm{~Hz}, 1 \mathrm{H}, \mathrm{C}_{3} \underline{\mathrm{H}}\right), 4.25(\mathrm{t}, J=$ $\left.6.6 \mathrm{~Hz}, 1 \mathrm{H}, \mathrm{C}_{4} \underline{\mathrm{H}}\right), 5.97\left(\mathrm{~s}, 2 \mathrm{H}, \mathrm{OC}_{2} \mathrm{O}\right), 6.76(\mathrm{~d}, J=5.9 \mathrm{~Hz}, 2 \mathrm{H}, \mathrm{Ar} \underline{\mathrm{H}}), 6.8$ (d, J = 6.6 Hz, 1H,

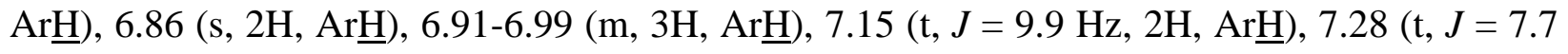
$\mathrm{Hz}, 1 \mathrm{H}, \mathrm{Ar} \underline{\mathrm{H}}), 10.55,10.58\left(2 \times \mathrm{s}, 2 \mathrm{H}\right.$, exchangeable with $\mathrm{D}_{2} \mathrm{O}, 2 \times \mathrm{N} \underline{\mathrm{H}}$ of isatyl ring and indole 
ring). ${ }^{13} \mathrm{C}$ NMR $\left(75 \mathrm{MHz}, \mathrm{DMSO}-d_{6}\right) \delta 39.8 ; 48.9,61.6,100.9,108.1,108.9,110.4,111.5,113.1$, $118.7,119.1,121.4,121.5,122.2$, 124.8, 126.3, 129.4, 132.8, 132.9, 136.7, 138.2 , 142.7, 146.1, 147.6, 178.4; $m / z: 409\left(\mathrm{M}^{+}\right)$. Anal. Calcd. for $\mathrm{C}_{25} \mathrm{H}_{19} \mathrm{~N}_{3} \mathrm{O}_{3}: \mathrm{C}, 73.34 ; \mathrm{H}, 4.68 ; \mathrm{N}, 10.26$. Found: C, 73.14; H, 4.89; N 10.01\%.

4-(2-Thienyl)-2,3,4,9-tetrahydrospiro[ $\boldsymbol{\beta}$-carboline-1,3'-indol]-2'(1'H)-one (37). Yield 81\%; brown solid; mp $134-136{ }^{\circ} \mathrm{C} ; \mathrm{R}_{\mathrm{f}}(50 \%$ ethyl acetate/hexane) 0.46 ; IR (KBr): v 3390, 3280, 3186, $1718 \mathrm{~cm}^{-1} .{ }^{1} \mathrm{H}$ NMR (300 MHz, DMSO-d 6 ) $\delta 2.96$ (br s, $1 \mathrm{H}$, exchangeable with $\left.\mathrm{D}_{2} \mathrm{O}, \mathrm{NH}\right), 3.44$ (br s, $\left.1 \mathrm{H}, \mathrm{C}_{3} \underline{\mathrm{H}}\right), 3.53\left(\mathrm{dd}, J=5.4,12.0 \mathrm{~Hz}, 1 \mathrm{H}, \mathrm{C}_{3} \underline{\mathrm{H}}\right), 4.62\left(\mathrm{t}, J=5.5 \mathrm{~Hz}, 1 \mathrm{H}, \mathrm{C}_{4} \underline{\mathrm{H}}\right), 6.8(\mathrm{t}, J=$ $7.6 \mathrm{~Hz}, 1 \mathrm{H}, \operatorname{Ar} \underline{\mathrm{H}}), 6.85-7.03$ (m, 6H, Ar$\underline{\mathrm{H}}), 7.15$ (t, $J=10.5 \mathrm{~Hz}, 2 \mathrm{H}, \operatorname{Ar} \underline{\mathrm{H}}), 7.19-7.3$ (m, 2H, $\operatorname{Ar} \underline{\mathrm{H}}), 10.58\left(\mathrm{~s}, 2 \mathrm{H}\right.$, exchangeable with $\mathrm{D}_{2} \mathrm{O}, 2 \times \mathrm{NH}$ of isatyl ring and indole ring). ${ }^{13} \mathrm{C} \mathrm{NMR}(75$ MHz, DMSO-d6) $\delta 35.1,48.8,61.1,109.8,111.0,112.6,118.2,118.4,120.9,121.6,123.7$, 124.3, 124.6, 125.6, 126.3, 128.9, 132.0, 132.4, 136.1, 142.1, 147.3, 177.6; m/z: $371\left(\mathrm{M}^{+}\right)$. Anal. Calcd. for $\mathrm{C}_{22} \mathrm{H}_{17} \mathrm{~N}_{3} \mathrm{OS}$ : C, 71.14; H, 4.61; N, 11.31. Found: C, 70.91; H, 4.79; N 11.12\%.

4-Phenyl-2,3,4,9-tetrahydrospiro[ $\boldsymbol{\beta}$-carboline-1,1'- $\boldsymbol{\alpha}$-tetralone] (38). Yield 76\%; white solid; mp $183-185^{\circ} \mathrm{C} ; \mathrm{R}_{\mathrm{f}}\left(50 \%\right.$ ethyl acetate/hexane) $0.50 ; \mathrm{IR}(\mathrm{KBr}): v 3437,3421 \mathrm{~cm}^{-1} .{ }^{1} \mathrm{H}$ NMR $(300$ $\left.\mathrm{MHz} \mathrm{CDCl}_{3}\right) \delta 1.87(\mathrm{~s}, 2 \underline{\mathrm{H}}), 2.05(\mathrm{~s}, 1 \underline{\mathrm{H}}), 2.17(\mathrm{~s}, 3 \underline{\mathrm{H}}), 3.81\left(\mathrm{dd}, J=6.6,13.2 \mathrm{~Hz}, 1 \mathrm{H}, \mathrm{C}_{3} \underline{\mathrm{H}}\right)$, 4.04 (dd, $\left.J=6.6,13.2 \mathrm{~Hz}, 1 \mathrm{H}, \mathrm{C}_{3} \underline{\mathrm{H}}\right), 4.4\left(\mathrm{t}, J=7.6 \mathrm{~Hz}, 1 \mathrm{H}, \mathrm{C}_{4} \underline{\mathrm{H}}\right), 5.71$ (br s, $1 \mathrm{H}$, exchangeable with $\left.\mathrm{D}_{2} \mathrm{O}, \mathrm{N} \underline{\mathrm{H}}\right), 6.95-7.05(\mathrm{~m}, 3 \mathrm{H}, \operatorname{Ar} \underline{\mathrm{H}}), 7.14(\mathrm{t}, J=7.7 \mathrm{~Hz}, 2 \mathrm{H}, \operatorname{Ar} \underline{\mathrm{H}}), 7.22(\mathrm{t}, J=7.7 \mathrm{~Hz}, 1 \mathrm{H}$, $\operatorname{Ar} \underline{\mathrm{H}}), 7.25-7.34(\mathrm{~m}, 6 \mathrm{H}, \operatorname{Ar} \underline{\mathrm{H}}), 7.42(\mathrm{~d}, J=7.7 \mathrm{~Hz}, 1 \mathrm{H}, \mathrm{Ar} \underline{\mathrm{H}}), 8.64$ (br s, 1H, exchangeable with $\mathrm{D}_{2} \mathrm{O}, \mathrm{NH}$ of indole ring). ${ }^{13} \mathrm{C} \mathrm{NMR}\left(75 \mathrm{MHz}, \mathrm{CDCl}_{3}\right) \delta 14.1,22.5$ (st.), 30.1, 36.7, 67.2, 113.6 (st.), 120.4 (st.), 122.5, 123.1, 124.7 (st.), 126.3, 126.5 (st.), 128.9, 129.0 (st.), 133.6 (st.), 135.4, 137.8; $m / z: 364\left(\mathrm{M}^{+}\right)$. Anal. Calcd. for $\mathrm{C}_{26} \mathrm{H}_{24} \mathrm{~N}_{2}$ : C, 85.68; H, 6.64; N, 7.69. Found: C, 85.35; H, $6.89 ; \mathrm{N}, 7.91 \%$.

4-(4-Methoxyphenyl)-2,3, 4,9-tetrahydrospiro[ $\boldsymbol{\beta}$-carboline-1,1'- $\boldsymbol{\alpha}$-tetralone] (39). Yield 72\%; oily; $\mathrm{R}_{\mathrm{f}}\left(60 \%\right.$ ethyl acetate/hexane) 0.45 ; IR (KBr): $v 3404,3302 \mathrm{~cm}^{-1} .{ }^{1} \mathrm{H}$ NMR (300 MHz, $\left.\mathrm{CDCl}_{3}\right) \delta 1.61(\mathrm{~s}, \underline{\mathrm{H}}), 1.91(\mathrm{~s}, 2 \underline{\mathrm{H}}), 2.2(\mathrm{~s}, 3 \underline{\mathrm{H}}), 3.72-3.89\left(\mathrm{~m}, 4 \mathrm{H}, \mathrm{C}_{3} \underline{\mathrm{H}}\right.$ and $\left.\mathrm{OC}_{3}\right), 4.03$ (dd, $J=$ 7.6, $\left.13.5 \mathrm{~Hz}, 1 \mathrm{H}, \mathrm{C}_{3} \underline{\mathrm{H}}\right), 4.38$ (t, $\left.J=7.6 \mathrm{~Hz}, 1 \mathrm{H}, \mathrm{C}_{4} \underline{\mathrm{H}}\right), 5.53$ (brs, $1 \mathrm{H}$, exchangeable with $\mathrm{D}_{2} \mathrm{O}$, $\mathrm{N} \underline{\mathrm{H}}), 6.81(\mathrm{t}, J=8.8 \mathrm{~Hz}, 3 \mathrm{H}, \operatorname{Ar} \underline{\mathrm{H}}), 6.95-7.07(\mathrm{~m}, 3 \mathrm{H}, \mathrm{Ar} \underline{\mathrm{H}}), 7.11-7.27$ (m, 4H, Ar$\underline{\mathrm{H}}), 7.34(\mathrm{~d}, J$ $=8.2 \mathrm{~Hz}, 1 \mathrm{H}, \operatorname{Ar} \underline{\mathrm{H}}), 7.42(\mathrm{~d}, J=7.6 \mathrm{~Hz}, 1 \mathrm{H}, \operatorname{Ar} \underline{\mathrm{H}}), 8.33$ (brs, $1 \mathrm{H}$, exchangeable with $\mathrm{D}_{2} \mathrm{O}, \mathrm{N} \underline{\mathrm{H}}$ of indole ring). ${ }^{13} \mathrm{C} \mathrm{NMR}\left(75 \mathrm{MHz}, \mathrm{CDCl}_{3}\right) \delta 14.0,22.3$ (st.), 27.6, 34.2, 54.7, 66.7, 113.6 (st.), 120.3 (st.), 121.4, 123.3, 124.0 (st.), 124.9 (st.), 126.6 (st.), 128.5, 129.1 (st.), 133.7 (st.), 135.2, 137.8, 155.1; m/z: $394\left(\mathrm{M}^{+}\right)$. Anal. Calcd. for $\mathrm{C}_{27} \mathrm{H}_{26} \mathrm{~N}_{2} \mathrm{O}: \mathrm{C}, 82.20 ; \mathrm{H}, 6.64 ; \mathrm{N}, 7.10$. Found: $\mathrm{C}$, 81.93; H, 6.89; N 7.33\%.

Compound (40). Yield 89\%; colorless crystal; mp 191-193 ${ }^{\circ} \mathrm{C} ; \mathrm{R}_{\mathrm{f}}(70 \%$ ethyl acetate/hexane) 0.11; IR (KBr): v 3367 (br) cm ${ }^{-1} .{ }^{1} \mathrm{H}$ NMR (300 MHz, DMSO-d 6 ) $\delta 1.52$ (s, 3H, $\mathrm{CH}_{3}$ ), 2.15-2.62 $\left(\mathrm{m}, 4 \mathrm{H},-\left(\underline{\mathrm{C}}_{2}\right)_{2}-\right), 3.85\left(\mathrm{dd}, J=6.4,13.8 \mathrm{~Hz}, 1 \mathrm{H}, \mathrm{C}_{3} \underline{\mathrm{H}}\right), 4.02\left(\mathrm{dd}, J=6.3,12.7 \mathrm{~Hz}, 1 \mathrm{H}, \mathrm{C}_{3} \underline{\mathrm{H}}\right)$, 4.49 (t, $J=7.2 \mathrm{~Hz}, 1 \mathrm{H}, \mathrm{C}_{4} \underline{\mathrm{H}}$ ), 6.63 (br s, $1 \mathrm{H}$, exchangeable with $\mathrm{D}_{2} \mathrm{O}, \mathrm{N} \underline{\mathrm{H}}$ ), 6.93 (t, $J=7.2 \mathrm{~Hz}$, $1 \mathrm{H}, \operatorname{Ar} \underline{\mathrm{H}}$ ), 7.09 (t, $J=7.2 \mathrm{~Hz}, 2 \mathrm{H}, \mathrm{Ar} \underline{\mathrm{H}}$ ), 7.13-7.50 (m, 7H, ArH $), 10.46$ (br s, 1H, exchangeable with $\mathrm{D}_{2} \mathrm{O}, \mathrm{NH}$ of indole ring). ${ }^{13} \mathrm{C}$ NMR $\left(75 \mathrm{MHz}, \mathrm{DMSO}-d_{6}\right) \delta 6.7,23.8,32.5,43.4,48.5$, $105.1,111.3,115.3,118.2,118.5,120.9$, 122.0, 126.1, 126.5, 128.0 (st.), 136.1, 143.1, 173.1, 
199.5; m/z: $330\left(\mathrm{M}^{+}\right)$. Anal. Calcd. for $\mathrm{C}_{22} \mathrm{H}_{22} \mathrm{~N}_{2} \mathrm{O}$ : C, 79.97; H, 6.71; N, 8.48. Found: C, 79.64; H, 6.99; N, 8.27\%.

\section{General procedure for preparation of compounds (33 and 34)}

A few drops of concentrated $\mathrm{H}_{2} \mathrm{SO}_{4}$ or $\mathrm{HCl}$ were added to compound $32(0.2 \mathrm{~g}, 0.55 \mathrm{mmol})$ dissolved in methanol $(3 \mathrm{~mL})$. The reaction mixture stirred with heating till the solution became clear and kept at room temperature for $24 \mathrm{~h}$. Completion of the reaction was confirmed by TLC. The solvent was removed and the crystals were washed twice with methanol to furnish the expected product 1,1-isatyl-4-phenyl-2,3,4,9-tetrahydrospiro- $\beta$-carboline sulfate $\mathbf{3 3}$ or hydrochloride 34 .

4-Phenyl-2,3,4,9-tetrahydrospiro[ $\beta$-carboline-1,3'-indol $]-2^{\prime}\left(1^{\prime} H\right)$-one sulfate $(33)$. Yield 95\%; colorless crystals; mp above $300{ }^{\circ} \mathrm{C}$; $\mathrm{R}_{\mathrm{f}}(50 \%$ acetone/hexane) 0.10; IR (KBr): $v$ 3389, 3244 (br), $1725 \mathrm{~cm}^{-1} .{ }^{1} \mathrm{H}$ NMR (300 MHz, DMSO- $\left.d_{6}\right) \delta 3.84\left(\mathrm{dd}, J=6.1,12.1 \mathrm{~Hz}, 1 \mathrm{H}, \mathrm{C}_{4} \underline{\mathrm{H}}\right)$, $4.14\left(\mathrm{t}, J=10.7 \mathrm{~Hz}\right.$, overlapped on DMSO- $\left.d_{6} 1 \mathrm{H}, \mathrm{C}_{3} \underline{\mathrm{Ha}}\right), 4.72\left(\mathrm{dd}, J=6.1,10.7 \mathrm{~Hz}, 1 \mathrm{H}, \mathrm{C}_{3} \underline{\mathrm{He}}\right)$, $6.54(\mathrm{~d}, J=7.7 \mathrm{~Hz}, 1 \mathrm{H}, \operatorname{Ar} \underline{\mathrm{H}}), 6.77$ (t, $J=7.2 \mathrm{~Hz}, 1 \mathrm{H}, \operatorname{Ar} \underline{\mathrm{H}}), 7.03$ (t, $J=8.3 \mathrm{~Hz}, 1 \mathrm{H}, \operatorname{Ar} \underline{\mathrm{H}}), 7.1$ $7.24(\mathrm{~m}, 3 \mathrm{H}, \operatorname{Ar} \underline{\mathrm{H}}), 7.32-7.45(\mathrm{~m}, 6 \mathrm{H}, \operatorname{Ar} \underline{\mathrm{H}}), 7.53(\mathrm{t}, J=7.7 \mathrm{~Hz}, 1 \mathrm{H}, \operatorname{Ar} \underline{\mathrm{H}}), 10.55,11.02$ and 11.41 (4x br s, $4 \mathrm{H}$, exchangeable with $\mathrm{D}_{2} \mathrm{O}, 2 \mathrm{xN \underline {H }}$ of isatyl ring and indole ring and $\mathrm{NH}_{2}$ ). ${ }^{13} \mathrm{C}$ NMR (75 MHz, DMSO- $\left.d_{6}\right) \delta 36.8,44.7,59.5,111.3,111.9$ (st.), 119.3 (st.), 122.4, 123.0, 124.4, $124.8,126.4,126.9,127.6,128.5$ (st.), 128.8 (st.), 132.1, 136.9, 140.4, 143.2, 172.1; $\mathrm{m} / \mathrm{z}: 435$ $\left(\mathrm{M}^{+}-28\right)$. Anal. Calcd. for $\mathrm{C}_{24} \mathrm{H}_{21} \mathrm{~N}_{3} \mathrm{O}_{5} \mathrm{~S}: \mathrm{C}, 62.19 ; \mathrm{H}, 4.57 ; \mathrm{N}, 9.07$. Found: $\mathrm{C}, 61.98 ; \mathrm{H}, 4.71 ; \mathrm{N}$ $8.81 \%$.

4-Phenyl-2,3,4,9-tetrahydrospiro[ $\boldsymbol{\beta}$-carboline-1,3'-indol $]-2^{\prime}\left(\mathbf{1}^{\prime} \boldsymbol{H}\right)$-one hydrochloride (34). Yield $94 \%$, colorless crystals; $m p \quad 255-257^{\circ} \mathrm{C} ; \mathrm{R}_{\mathrm{f}}(90 \%$ ethyl acetate/hexane) 0.40 ; IR (KBr): $v$ 3306, 3248, 3217 (br), $1739 \mathrm{~cm}^{-1} .{ }^{1} \mathrm{H}$ NMR (300 MHz, DMSO-d $) \delta 3.71$ (dd, $J=6.1,12.1 \mathrm{~Hz}$, $\left.1 \mathrm{H}, \mathrm{C}_{4} \underline{\mathrm{H}}\right), 4.17\left(\mathrm{t}, J=11.3 \mathrm{~Hz}, 1 \mathrm{H}, \mathrm{C}_{3} \underline{\mathrm{Ha}}\right), 4.83\left(\mathrm{dd}, J=6.1,10.6 \mathrm{~Hz}, 1 \mathrm{H}, \mathrm{C}_{3} \underline{\mathrm{He}}\right), 6.54(\mathrm{~d}, J=8.0$ $\mathrm{Hz}, 1 \mathrm{H}, \operatorname{Ar} \underline{\mathrm{H}}), 6.76(\mathrm{t}, J=7.4 \mathrm{~Hz}, 1 \mathrm{H}, \operatorname{Ar} \underline{\mathrm{H}}), 7.02$ (t, $J=7.7 \mathrm{~Hz}, 1 \mathrm{H}, \operatorname{Ar} \underline{\mathrm{H}}), 7.13$ (t, $J=7.7 \mathrm{~Hz}$,

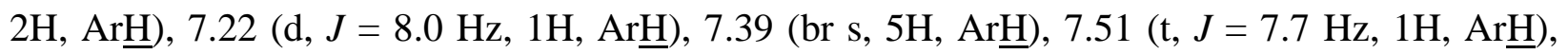
$7.63(\mathrm{~d}, J=7.4 \mathrm{~Hz}, 1 \mathrm{H}, \mathrm{Ar} \underline{\mathrm{H}}), 10.97,11.4$ (2x br s, 4H, exchangeable with $\mathrm{D}_{2} \mathrm{O}, 2 \mathrm{xN} \underline{\mathrm{H}}$ of isatyl ring and indole ring and $\left.\underline{\mathrm{N}}_{2}\right) .{ }^{13} \mathrm{C} \mathrm{NMR}\left(75 \mathrm{MHz}, \mathrm{DMSO}-d_{6}\right) \delta 38.8,48.3,60.9,109.7,111.0$, 112.6, 118.0, 118.5, 120.7, 121.5, 124.4, 125.6, 126.0, 127.9(st.), 128.0 (st.), 128.9, 132.2, 132.5, 136.0, 142.2, 143.6, 177.8; m/z: $365\left(\mathrm{M}^{+}-36\right)$. Anal. Calcd. for $\mathrm{C}_{24} \mathrm{H}_{20} \mathrm{ClN}_{3} \mathrm{O}: \mathrm{C}, 71.73 ; \mathrm{H}, 5.02$; N, 10.46. Found: C, 71.52; H, 4.81; N, 10.22\%.

\section{Crystallography}

Crystallographic data in this paper have been deposited with the Cambridge Crystallographic Data Centre. Deposition numbers are CCDC 752864 for 33, CCDC 752865 for 40, CCDC 752866 for 32 and CCDC 752867 for 34. Copies of the data can be obtained, free of charge, on application to CCDC, 12 Union Road, Cambridge CB2 1EZ, UK (fax: +44 1223336033 or email: deposit@ccdc.cam.ac.uk.

Crystal data. The X-ray data of all the four compounds were collected at $T=296 \mathrm{~K}$, on SMART APEX CCD Single Crystal X-ray diffractometer using Mo-K $\alpha$ radiation $(\lambda=0.7107 \AA)$ to a 
maximum $\theta$ range of $25.00^{\circ}$. The structures were solved by direct methods using SHELXTL. All the data were corrected for Lorentzian, polarization and absorption effects. SHELX-97 $\left(\right.$ ShelxTL) ${ }^{28}$ was used for structure solution and full matrix least squares refinement on $\mathrm{F}^{2}$. Hydrogen atoms were included in the refinement as per the riding model. The refinements were carried out using SHELXL-97.

\section{Acknowledgements}

We are grateful to Prof. M. S. Wadia for helpful discussion, B. S. Kalshetti for IR spectra, J. P. Choudhary for NMR spectra, and D. S. Shishupal for GCMS. A.M.A.S. is thankful to MOHE in Yemen for the award of a fellowship.

\section{References}

1. Pogosyan, S. A.; Grigoryan, N. P.; Paronikyan, R. G. Pharm. Chem. J. 2007, 41, 527.

2. Grigoryan, N. P.; Pogosyan, S. A.; Paronikyan, R. G. Hayastani Kimiakan Handes 2005, 58, 100.

3. Peter, B.; Ulf, B.; Roger, C.; Malin, G. N.; Annika, J. J.; Erik, R.; Terry, W. PCT Int. Appl. WO 2005048 916, 2005, 153.

4. Balsamini, C.; Spadoni, G.; Duranti, E.; Cerrini, S.; Lamba, D.; Fedeli, W. Farmaco 1989, 44, 399.

5. Synthelabo, S. A. Fr. Jpn. Kokai Tokkyo Koho. JP 55145687, 1980, 9.

6. Pictet, A.; Spengler, T. Chem. Ber.1911, 44, 2030.

7. (a) Bringmann, G.; Ewers, C. T.; Walter, R. Comprehensive Organic Synthesis; Trost, B. M.; Fleming, I., Eds.; Pergamon Press: Oxford, 1991; Vol. 6, pp 736-740. (b) Yoshie, H.; Masayoshi, N.; Toshiaki, S.; Takehiro, S. Chem. Pharm. Bull. 2003, 51, 1368.

8. Cox, E. D.; Cook, J. M. Chem. Rev. 1995, 95, 1797.

9. Chrzanowska, M.; Rozwadowska, M. D. Chem. Rev. 2004, 104, 3341.

10. Seayad, J.; Seayad, A. M.; List, B. J. Am. Chem. Soc. 2006, 128, 1086.

11. Taylor, M. S.; Jacobsen, E. N. J. Am. Chem. Soc. 2004, 126, 10558.

12. Alberch, L.; Bailey, P. D.; Clingan, P. D.; Mill, T. J.; Price, R. A.; Pritchard, R. G. Eur. J. Org. Chem. 2004, 1887.

13. Tsuji, R.; Nakagawa, M.; Nishida, A. Tetrahedron: Asymmetry 2003, 14, 177.

14. Yu, J.; Wearing, X. Z.; Cook, J. M. Tetrahedron Lett. 2003, 44, 543.

15. Bonnet, D.; Ganesan, A. J. Comb. Chem. 2002, 4, 546.

16. Bailey, P. D.; Hollinshead, S. P.; Malcy, N. R. Tetrahedron Lett. 1987, 28, 5177.

17. Sandrin, J.; Hollinshead, S. P.; Cook, J. M. J. Org. Chem. 1989, 54, 5636.

18. Singh, K.; Deb, P. K. Tetrahedron Lett. 2000, 41, 4977. 
19. Singh, K.; Deb, P. K.; Venugopalan, P. Tetrahedron 2001, 57, 7939.

20. Jacobsen, E. N.; Klausen, R. S. Org. Lett. 2009, 11, 887.

21. Gremmen, C.; Willemse, B.; Wanner, M. J.; Koomen, G.-J. Org. Lett. 2000, 2, 1955.

22. Larghi, E. L.; Amongero, M.; Bracca, A. B. J.; Kaufman, T. S. Arkivoc 2005, (xii), 98.

23. (a) Semenov, B. B.; Novikov, K. A.; Spitsin, A. N.; Azev, V. N.; Kachala, V. V. Chem. Nat. Comp. 2004, 40, 585. (b) Semenov, B. B.; Novikov, K. A.; Smushkevich, Y. I.; Azev, V. N.; Kachala, V. V. Chem. Heterocycl. Compd. 2005, 41, 1273. (c) Herrera, R. P.; Sgarzani, V.; Bernardi, L.; Ricci, A. Angew. Chem., Int. Ed. 2005, 44, 6576.

24. Semenov, B. B.; Novikov, K. A.; Azev, V. N.; Kachala, V. V. Russ. Chem. Bull. Int. Ed. 2005, 54, 988.

25. (a) Kusurkar, R. S.; Alkobati, N. A.; Gokule, A. S.; Puranik, V. G. Tetrahedron 2008, 64, 1654. (b) Busacca, C. A.; Dong, Y. Synth. Commun. 2000, 30, 501.

26. (a) Coma, A. Chem. Rev. 1995, 95, 559. (b) Isobe, K. Acc. Chem. Res. 1993, 26, 524.

27. (a) Kamitori, Y.; Hojo, M.; Masuda, R.; Izumi, T.; Tsukamoto, S. J. Org. Chem. 1984, 49, 4161. (b) Kotsuki, H.; Hayashida, K.; Shimanouchi, T.; Nishizawa, H. J. Org. Chem. 1996, 61, 984. (c) Chavan, F.; Madje, B.; Bharad, J.; Ubale, M.; Ware, M.; Shingare, M.; Shinde, N. Bull. Cat. Soc. India 2008, 7, 41. (d) Kusurkar, R. S.; Alkobati, N. A. H.; Gokule, A. S.; Chaudhari, P. M.; Waghchaure, P. B. Synth. Commun. 2006, 36, 1075.

28. Sheldrick, G. M. SHELX-97, Program for Crystal Structure Solution and Refinement; University of Göttingen: Göttingen, Germany, 1997. 